\title{
Notice bio-bibliographique de Pierre Vernet
}

Piblikasyon sa a reprezante yon omaj pou Pierre Vernet, youn nan pi gwo lengwis ayisyen ki mouri nan tranbleman tè 12 janvye 2010 la, nan lokal Fakilte Lengwistik Aplike (FLA) an Ayiti. Pierre Vernet te devlope relasyon pandan lontan ak kòlèg ak zanmi li yo ki travay nan diferan domèn lengwistik. Se sa ki fè dosye sa a ap gen atik sou lang ak literati kreyòl, pa sou kreyòl ayisyen ase ni kreyòl zantiy yo. Pierre Vernet te travay anpil tou ak diferan lòt kòlèg sou kesyon amenajman lengwistik, plas lengwistik nan diferan domèn sosyal, òganizasyon filyè bileng ak frankofoni.

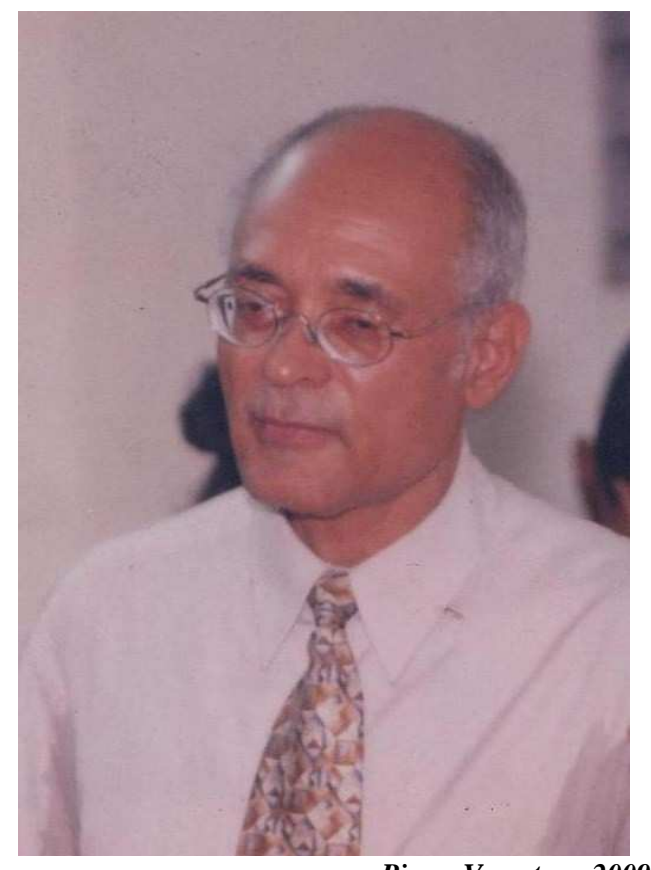

Pierre Vernet, en 2009

Frédéric Torterat, Université de Nice

Pierre Vernet est né le 21 mars 1943 à Lascaobas, dans le Centre d'Haïti, entre Mirebalais et Belladère. Ses parents s'étant rendus très tôt dans la capitale, il a effectué ses études primaires à l'Ecole congréganiste Jean-Marie Guilloux, à Port-auPrince, entre la rue Pavée et la Grand-Rue. Pierre Vernet accomplit sa scolarité du secondaire au Petit Séminaire Saint-Martial, rue Lamarre, et obtient son baccalauréat, mention philosophie, en 1964. C'est alors que commence pour lui une carrière d'enseignant (de français, anglais et latin), dans différents établissements haïtiens d'abord (1964-1966), puis aux Etats-Unis, de 1966 à 1970, où il participe à plusieurs campagnes d'alphabétisation d'enfants et d'adolescents de populations défavorisées.

Son parcours universitaire le conduit à Paris V, où il devient l'étudiant d'André 
Martinet et de Louis-Jean Calvet, pour un premier cycle en Psychologie et en Linguistique (1970-1973), auquel fera suite un deuxième cycle en Linguistique et Sciences de l'éducation (1974-1976). Ces cursus doubles l'inciteront à poursuivre ses recherches doctorales dans les mêmes domaines, toujours à Paris V, jusqu'à l'obtention de son doctorat de linguistique créole en 1984. Entre temps, Pierre Vernet s'implique activement dans son pays natal, où il donne des cours de méthodologie de l'enseignement dans les Ecoles Normales et à la Faculté d'Agronomie, et où il anime divers stages auprès d'enseignants d'écoles publiques et privées, dans le primaire comme dans le secondaire. Les mandats qui lui ont été confiés par le Ministère de l'Education nationale haïtienne et l'Institut Pédagogique National l'amènent respectivement à contribuer à la politique linguistique du pays, et à participer à la Réforme du système éducatif national, à travers en particulier l'organisation de nouveaux curriculums.

C'est dans ce contexte, à la faveur d'une recherche doctorale de terrain et avec le soutien notamment du linguiste haïtien Pradel Pompilus, que Pierre Vernet créera, en 1978, la Faculté de Linguistique Appliquée de Port-au-Prince. Il le fera d'abord avec l'aide de l'AUPELF (AUF), ainsi que d'autres universités partenaires, comme l'Université des Antilles et de la Guyane (UAG) et Paris V. Celui qui sera bientôt Doyen de la FLA multipliera les collaborations et les partenariats de manière à consolider peu à peu une institution qui formera, jusqu'en 2009, des milliers d'étudiants venus de tout le pays, et ce pour de nombreux corps de métiers. Bientôt Pierre Vernet devient le correspondant national de la CONFEMEN (Conférence des Ministres de l'Education Nationale des pays d'Expression Française), et celui du réseau «Industrie de la langue » dans le cadre du Sommet Francophone. Dans le même temps, il contribue à la mise au point d'une graphie officielle du créole haïtien (en rapport avec ses recherches en phonologie et en techniques d'écriture), et travaille également aux Seychelles au moment des principales réformes éducatives. En lien avec la valorisation des produits de cette recherche, Pierre Vernet, tout en participant activement à la nouvelle collection Espaces créoles, s'implique personnellement dans Bannzil Kreyol, une association de promotion de la langue créole qu'il soutient dès le début, en 1981, quand il en est question pour la première fois en marge du Colloque international des Etudes créoles à Sainte-Lucie. Il supervisera, en outre, la rédaction 
de plusieurs dictionnaires, glossaires et terminologies bilingues, qui trouveront des aboutissements concrets dans la production de livrets d'alphabétisation sollicités dans toutes les régions du pays. En charge, enfin, des relations avec l'OIF au titre notamment de la didactique adaptée du français en milieu de créolophonie, et avec la FIPF en Haïti, Pierre Vernet consent à accompagner de nombreux projets, ce qui conduira Abdou Diouf, Secrétaire général de la Francophonie, à lui remettre la plaque d'honneur et du mérite le 21 mars 2005.

En tous temps et malgré les tentatives d'intimidation dont il a fait quelquefois l'objet, Pierre Vernet a poursuivi continument ses efforts dans un esprit d'indépendance et de lutte pour la langue créole, mais aussi pour une liberté d'expression qui l'a mis plusieurs fois en péril. C'est dans un esprit aussi combatif qu'il pérennisera la Faculté de Linguistique Appliquée, avec une consolidation sans cesse maintenue de ses équipes pédagogiques, de sa recherche et de ses spécificités. Très investi dans les questions d'aménagement linguistique, aussi sensible à l'existant qu'à l'innovation, ne cédant ni à l'improvisation des discours, ni à la politique du moment, Pierre Vernet amènera la FLA à nouer des relations durables avec les Universités, parmi d'autres, de Bruxelles, Grenoble, La Havane, Louvain, Mons, Nice, ainsi que plusieurs UFR parisiennes.

Les travaux universitaires de Pierre Vernet s'inscrivent principalement dans le domaine de la linguistique, et portent notamment sur les marques TMA (temps, mode, aspect), la prédicativité, l'indéfini, au fil d'une réflexion qui l'a amené à s'interroger en outre sur les langues indonésienne(s) et bambara. Pierre Vernet s'est par ailleurs penché très tôt sur des questions d'ordre sociolinguistique (à partir de l'apprentissage de l'anglais dans la communauté haïtienne de New York) et psycho-pédagogique (à travers les formes d'apprentissage de l'haïtien et du français en Haïti, mais aussi les places de l'enfant et de l'adolescent dans le cercle familial).

Les travaux de Pierre Vernet dans le domaine de la syntaxe se sont d'abord concentrés sur les prédicats en créole haïtien et plus spécifiquement sur la prédicativité des arguments verbaux (1973), une démarche qu'il a menée presque simultanément dans une perspective typologique avec le bambara (1975). Peu après, Pierre Vernet a appliqué son analyse des structures linguistiques aux conditions de 
leur appropriation par divers apprenants, ce qui l'a conduit à rapporter bientôt cette recherche à des enjeux d'alphabétisation (1979, 1981), de didactisation (1980b, 1983) et d'éducation (1984a, b, 1989b). Les aspects plus particulièrement méthodologiques et sociolinguistiques de l'enseignement du créole haïtien vont ainsi l'occuper dès sa recherche doctorale, et ce durant toute sa carrière. Ainsi envisagera-t-il ces questions à l'appui de la phonologie et de la morphologie (1980a, 1981, 1988), de la syntaxe (1984b, 1991, 2008a), mais également de la terminologie (1990b, 2001a), de l'aménagement linguistique (1984a, 1990a, 2008b) ainsi que des processus de (trans-)continuum dont rend compte la créolistique (1989a, 2006).

Pierre Vernet a surtout constamment milité pour une reconnaissance de la justesse et de la productivité néologiques de l'haïtien dans tous les secteurs de la demande sociale, ce dont les dictionnaires et les terminologies qu'il a supervisés avec autant d'intransigeance que d'élégance témoignent au premier plan (1976, 1988, 1989a, 2001a). Pierre Vernet n'a d'ailleurs pas hésité à le faire à l'appui de la littérature, comme il s'y est employé dans l'un de ses derniers ouvrages, consacré au roman Eritye Vilokan de Pierre Michel Chéry (2001b), et où il écrit discrètement ceci :

Lorsqu'un être humain parle, ce qu'il exprime dit ce qu'il est. Qu'est-ce qu'il est ? Le résultat de sa propre vie, de ce qu'il a vécu. Une vie qui prend corps dans le vivre avec les autres, une vie qui s'enracine dans la nuit des temps et qui vient s'actualiser dans le brassage avec les faits d'aujourd'hui.

En décembre 2009, lors de notre dernière semaine passée ensemble à Port-auPrince, Pierre Vernet nous a énuméré ses nombreux projets. Parmi eux, un Dictionnaire trilingue créole haïtien / français / anglais, un Lexique de technologie, une Linguistique bilingue du créole haïtien, ainsi que plusieurs ouvrages didactiques menés en collaboration.

Le séisme de janvier 2010 l'a emporté brusquement, comme les nombreux étudiants qui suivaient son cours, au deuxième étage de la FLA.

Le 12 janvier 2010. 


\section{Principales publications de Pierre Vernet (hors articles journalistiques) :}

- (1973). Analyse Syntaxique du Créole Haïtien : Prédicat, Prédicatifs et nonprédicatifs. Université de Paris V, mémoire de premier cycle en syntaxe créole.

- (1975). Une Analyse syntaxique du Bambara. Université de Paris V, mémoire de master.

- Avec P. Nougayrol et A. Bentolila (1976). Ti diksyonnè kreyòl-franse. Port-auPrince, éditions Caraïbes.

- (1979). Alphabétisation en Haïti : aspect linguistique. Gerec 4, Centre Universitaire Antilles-Guyane, Editions Caribéennes.

- (1980a). Techniques d'écriture du créole haïtien, Port-au-Prince, Le Natal.

- (1980b). Le créole haïtien face à son introduction en salle de classe : le champ sémantique du corps humain. Etudes créoles 3/2 : 45-55.

- (1981). L'écriture du créole et ses réalités de fonctionnement. Cahiers du Centre de Linguistique appliquée 3 : 1-19.

- Avec R. Chaudenson (1983). L'école en créole. Etude comparée des réformes des systèmes éducatifs en Haïti et aux Seychelles. Paris, ACCT.

- (1984a). Langues, éducation et société en Haïti. Université de Paris V, thèse de Doctorat de 3e cycle (2 tomes), $661 \mathrm{p}$.

- (1984b). La reforme éducative en Haiti. Philosophie, objectifs, stratégies et contenus. Études créoles 7/1-2.

- Avec B.C. Freeman (1988). Diksyonè òtograf kreyòl ayisyen. Port-au-Prince, Sant Lengwistik aplike, Inivèsite Leta Ayiti.

- Avec B.C. Freeman (1989a). Dictionnaire préliminaire des Fréquences de la langue créole haïtienne. Port-au-Prince, Sant Lengwistik aplike, Inivèsite Leta Ayiti.

- (1989b). Hommage au Dr. Pradel Pompilus. Port-au-Prince, Sant Lengwistik aplike, Inivèsite Leta Ayiti.

- (1990a). L'enseignement du français en milieu créolophone haïtien. Quelques aspects sociolinguistiques et méthodologiques. Espace créole 7 : 98-149.

- (1990b). Problématique de la recherche terminologique en Haïti. Terminologies nouvelles (Cahiers du Rifal), 3.

- Avec H. Wittmann et R. Fournier (1991). Syntaxe descriptive et comparée du créole haïtien. Conseil de recherches en sciences humaines du Canada. 
- Avec H. Tourneux (dir.) (2001a). Leksik elektwomekanik kreyòl, franse, angle, espayòl. Port-au-Prince, Fakilte Lengwistik Aplike, Inivèsite Leta Ayiti.

- (2001b). Analyse du roman / Analiz woman Eritye Vilokan, de Pierre Michel Chéry. Port-au-Prince, Anthropos.

- (2006). Trente ans de créolistique. Processus de re-créolisation, décréolisation. In E. Dorismond, F. Calixte et N. Santamaria (éds.), La Caraïbe, entre Histoire et Politique (Recherches Haïtiano-antillaises 4), Paris, L'Harmattan.

- (2008a). Une Démarche d'adaptation de la didactique du français aux enfants créolophones. In R. Chaudenson (dir.), Didactique $d u$ français en milieux créolophones. Outils pédagogiques et formation des maîtres, Paris, L'Harmattan : 249-267.

- Avec L.J. Calvet, R. Chaudenson, J.L. Chiss \& C. Noyau (2008b). L'enseignement $\mathrm{du}$ français au primaire dans les aires créolophones. Actes du 12ème Congrès de la FIPF, Québec (Faire vivre les identités francophones) : 1209-1212. 